\title{
Guttural pouch equine: topography and vascularization
}

\author{
Nikolai Zelenevsky, Alexey Prusakov, Anatoly Yashin, Varvara Karanina, and Vladimir \\ Ponamarev $^{*}$ \\ Federal State Budgetary Educational Institution of Higher Education "St. Petersburg State University \\ of Veterinary Medicine", Department of Animal Anatomy, Chernigovskaya st. 5, 196084, St. \\ Petersburg, Russia
}

\begin{abstract}
The guttural pouch is a diverticulum of the auditory tube, the function of which hasn't been fully understood yet. The work reveals the anatomy and topography of the guttural pouch. This knowledge is necessary for the treatment of mycosis of the guttural pouch, disease leading to toxic hepatitis. The examination includes postmortem dissection, sagittal head section, and cerebral bilateral angioradiography. The auditory tube diverticulum is a complex anatomical structure, consisting of a volumetric cavity, divided by the stylohyoid into rostral and caudal sections. Large maxillary, external and internal carotid arteries, some cranial nerves pass along the lateral wall of the diverticulum. The tissues of the wall of the diverticulum are affected by the angiotropic fungus Aspergillus fumigatus. The main role of the studied arteries in mycosis of the guttural pouch wall is to provide the pathogenic fungus with nutrients, which leads to destruction of vascular wall and lethal epistaxis. The placement of arteries near the most important cranial nerves causes the damage of them and the development of severe neurological disorders. The diameter of the arteries determines the choice of occlusive material for the surgical treatment of mycosis. Latin anatomical terminology corresponds to the 6th edition of the International Veterinary Anatomical Nomenclature.
\end{abstract}

\section{Introduction}

Horses have a unique anatomical structure - the auditory tube diverticulum (diverticulum tubae auditivae), or guttural pouch. Its function hasn't been fully determined yet. Moreover, its wall is often affected by pathogenic fungi that cause its perforation and destruction of the membranes of the adjacent large arteries. This can lead to the death of the horse. Detailed and accurate knowledge of the anatomy of the guttural pouch, including the adjacent arteries and nerves, is necessary for a veterinarian-hippologist for the diagnosis and treatment of fungal lesions of this organ [1, 2, 3, 4], as well as for veterinary hepatologists, since as a result of hematogenous dissemination of mycoses pathogens primarily affects the liver [5], which leads to toxic hepatitis [6].

\footnotetext{
* Corresponding author: psevdopyos@mail.ru
} 
The study aimed to consider the location of the diverticulum of the auditory tube (guttural pouch) of a horse, its structure, and topography, including arterial vascularization.

\section{Materials and methods}

The study was carried out at the Department of Animal Anatomy at the St. Petersburg State University of Veterinary Medicine. The object of the study was the head of a Hanoverian horse. Within the framework of this work, postmortem bilateral angioradiography of the horse's head with the preliminary injection of the arterial bed with a radiopaque solidifying mass was developed and performed for the first time. It consisted of lead salts suspended in a solvent.

The arterial bed was infused with a radiopaque substance of an isolated horse's head through the common carotid arteries. The quality of the injection was assessed by the fullness of the vessels of the conjunctiva, the mucous membrane of the tongue, and auricular arteries. Subsequently, the object was prepared for bilateral angiography. This process requires the researcher to have a deep knowledge of anatomy and volumetric syntopy of the horse's head organs. It consists of an incomplete dorsoventral dissection of the organs of the head and neck of the animal in a strictly median plane. In this case, the organs of the intermaxillary space, larynx, trachea and esophagus are left intact. With the help of Liston bone forceps, the mandibles are divided along the mandibular suture and moved laterally. This material preparation algorithm achieves the possibility of bilateral Xray visualization of organs and extramural blood vessels of the horse's head without imposing "shadows" of symmetric structures of the same name. Radiography was performed on an electron matrix with subsequent morphometry.

A total of 11 horses of the Hanoverian breed, aged from 10 to 25 years, were examined. Cadaver material was obtained from animals that died as a result of accidents and diseases not associated with damage to the organs of the head and neck. The studies were carried out following the requirements of the European Convention for the Protection of Animals Used for Scientific Purposes.

\section{Results}

The guttural pouch (diverticulum tubae auditivae) of the horse is located under the bones of the base of the skull at the level from the caudal contour of the ethmoid labyrinth to the cranial edge of the atlas. Its cavity has a volume of $472.0 \pm 42.4 \mathrm{ml}$ and is filled with air when inhaled through the pharyngeal opening of the auditory tube (ostium pharyngeum tubae auditivae), which is covered with a fibro-cartilaginous plate (torus tubarius). Since the hole is located in the upper third of the air sac, the outflow of secretion from it is possible only when the horse's head is lowered.

The wall of the pouch is represented by a connective tissue plate lined with ciliated epithelium. Laterally adjacent to the wall are large vascular collectors: internal carotid, external carotid, and maxillary arteries. The difficulty of the secretion outflow, the proximity of the arteries and the thin wall of the diverticulum cause mortal danger to the horse's health when aggressive microorganisms, such as fungi, enter its cavity.

Mycosis of the guttural pouch is an infectious chronic disease of horses, characterized by unilateral nasal discharge and bleeding, various neuropathies. The causative agent is the angiotropic fungi Aspergillus fumigatus. Their spores are ubiquitous, including forage. Sports horses are especially sensitive to them. The nutrition of the fungus that has settled in the wall of the guttural pouch is carried out due to its tissues and adjacent arteries. This is accompanied by their destruction, leading to severe bleeding with a fatal outcome. The 


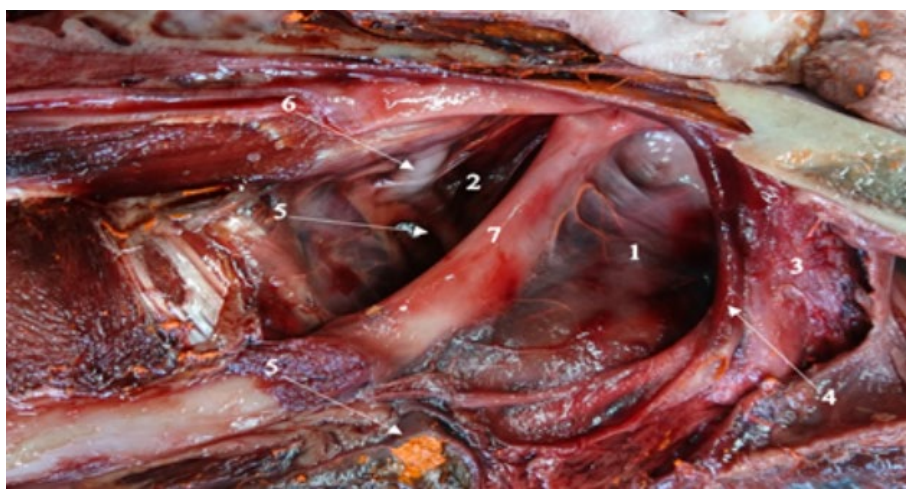

Fig. 1. Guttural pouch (diverticulum tubae auditivae) (lateral wall removed): 1 - medial and 2 - lateral sections of the guttural pouch; 3 - fold of the mucous membrane; 4 - internal carotid artery; 5 external carotid artery; 6 - maxillary artery; 7 - stylohyoid.

defeat of the nerves located near the arteries causes the following symptoms: Horner's syndrome, dysphagia, ataxia, respiratory murmurs.

On the medial side of the median cut of the horse's head, the guttural pouch is located dorsally from the nasopharynx, ventrally under the cerebellum, caudally from the ethmoid labyrinth, and cranially from the atlas. Rostrally, the pouch is bordered by the body of the sphenoid bone. Ventrally, it is in contact with the pharynx, esophagus, and retropharyngeal medial lymph nodes. Adjacent to the pouch is the digastric muscle, parotid, and mandibular glands. Dorsally from it lies the stony part of the temporal bone, the tympanic bladder, and the external auditory canal.

The middle segment of the hyoid bone divides the sac cavity 3: 1 into medial and lateral sections. The external carotid artery is adjacent to the lateral wall of the sac, and its branch, the maxillary artery, runs along the dorsal wall of the organ (Figure 1,2). In the wall of the caudal part of the medial part of the diverticulum, there is a fold of the mucous membrane. It contains the internal carotid artery, the cervical part of the sympathetic trunk, glossopharyngeal, accessory, and hypoglossal nerves.

On a digital bilateral vasoradiogram, we clarified the topography of the internal carotid, external carotid, and maxillary arteries, and using special software we measured the diameter of these vessels and their branches.

The common carotid artery $(10.30 \pm 0.98 \mathrm{~mm}$ : hereinafter, the diameter of the artery is given in $\mathrm{mm}$ ), lies on the dorsolateral surface of the trachea. At the level of the occipitalatlas joint, it gives off a branch of the internal carotid artery and continues as the external carotid artery. The internal carotid artery $(4.31 \pm 0.41 \mathrm{~mm})$ inside the mucous fold, with the IX, X, and XII cranial nerves, goes along the caudal wall of the guttural pouch.

The occipital artery $(5.30 \pm 0.49 \mathrm{~mm})$ emerges from the external carotid distal $1 \mathrm{~mm}$ from the internal carotid artery, accompanies it ventrolaterally for $2 \mathrm{~cm}$ in the rostral direction, and then bends dorsally at a $90^{\circ}$ angle. In horses, the occipital and internal carotid artery can branch off from the common carotid artery as a single trunk. The external carotid artery $(9.51 \pm 0.91 \mathrm{~mm}$ after the lingo-facial trunk is $6.25 \pm 0.59 \mathrm{~mm})$, passes ventrolaterally along the wall of the guttural pouch. It goes under the branch of the lower jaw in the cranioventral direction, it gives off a branch of the lingo-facial trunk. Then the magistral vessel goes dorsally along the lateral wall of the guttural pouch and after branching with one trunk, less often in series, the caudal auricular $(4.53 \pm 0.42 \mathrm{~mm})$ and superficial temporal arteries $(2.76 \pm 0.25 \mathrm{~mm})$, the external carotid artery bends almost under $90^{\circ}$ angle, changing the name to the maxillary artery $(4.75 \pm 0.45 \mathrm{~mm})$. It passes along the laterodorsal surface of the air sac in the rostral direction, next to the VII and VIII cranial nerves. 


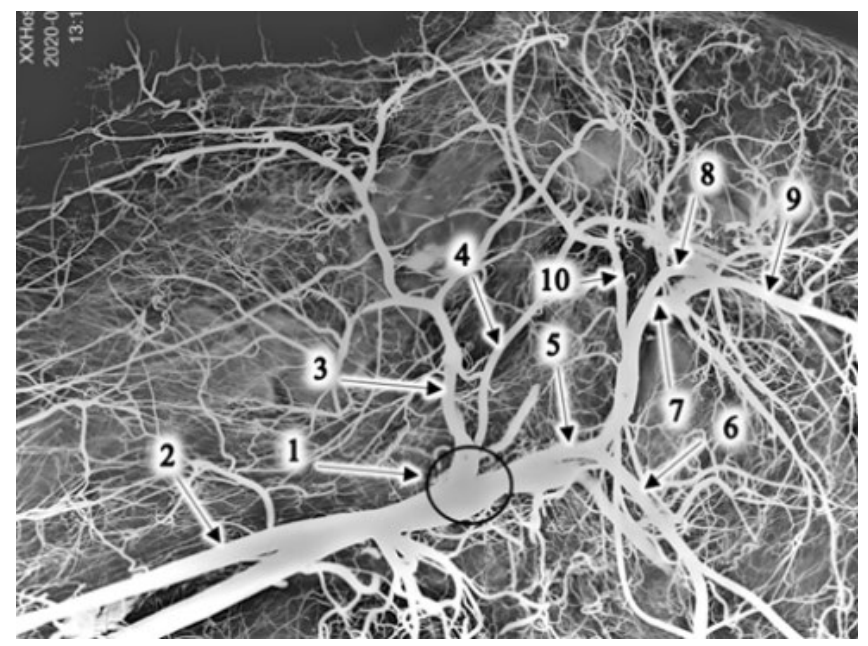

Fig. 2 - Common carotid artery and its branches of the horse. Vasoradiogram, lateral projection: 1 trifurcation of the carotid arteries; 2 - common carotid artery; 3 - occipital artery; 4 - internal carotid artery; 5 - external carotid artery; 6 - lingual-facial trunk; 7 - maxillary artery; 8 - transverse artery of the face; 9 - infraorbital artery; 10 - common trunk of the caudal auricular and superficial temporal arteries.

\section{Conclusion}

The auditory tube diverticulum is a complex anatomical structure consisting of two divisions separated by the stylohyoid, along the walls of which large arteries and cranial nerves pass.

The diameter of the internal carotid artery is $4.31 \pm 0.41 \mathrm{~mm}$; external carotid $-9.51 \pm$ $0.91 \mathrm{~mm}$, and maxillary $-4.75 \pm 0.45 \mathrm{~mm}$.

The main role of the studied arteries in mycosis of the guttural pouch is to provide the pathogenic fungus with nutrients, which grows through and causes destruction of the vascular wall and lethal epistaxis. The placement of arteries near the most important cranial nerves causes damage to them and the development of severe neurological disorders. The anatomy of the arteries of the guttural pouch area plays a direct role in the treatment of mycosis. The branching of the arteries leads to the formation of vascular anastomoses, which, in the event of artificial overlap, provide retrograde blood flow, due to which the animal does not recover. The diameter of the arteries determines the choice of occlusive material for the surgical treatment of mycosis. Thus, the veterinarian-hippologist needs to be aware of all the anatomical features of the arteries in the area of the guttural pouch.

\section{References}

1. N.V. Zelenevsky, Equine Anatomy, 405-412, (2018)

2. S. Reed, W. Bayly, D. Sello, Equine Internal Medicine, 4th edition, 325-327 (USA: Elsevier, 2018)

3. J. Auer, J. Stick, Equine Surgery, 5th edition, 777-780 (USA: Elsevier, 2019)

4. A. Ludwig, S. Gatineau, M-C Reynaud, et al., The veterinary journal 169 (3), 457-461 (2005) https://www.sciencedirect.com/science/article/abs/pii/S1090023304001388?v ia\%3Dihub 
5. V. Ponamarev, O. Popova, Issues of legal regulation in veterinary 3, 124-125 (2020) https://www.elibrary.ru/item.asp?id=44044368

6. V. Ponamarev, International bulletin of veterinary 2, 116-121 (2020) https://www.elibrary.ru/item.asp?id=43860222 